\title{
On Combining Lensing Shear Information from Multiple Filters
}

\author{
Mike Jarvis and Bhuvnesh Jain \\ Dept. of Physics and Astronomy, University of Pennsylvania, Philadelphia, PA 19104 \\ mjarvis, bjain@physics.upenn.edu
}

\begin{abstract}
We consider the possible gain in the measurement of lensing shear from imaging data in multiple filters. Galaxy shapes may differ significantly across filters, so that the same galaxy offers multiple samples of the shear. On the other extreme, if galaxy shapes are identical in different filters, one can combine them to improve the signal-to-noise and thus increase the effective number density of faint, high redshift galaxies. We use the GOODS dataset to test these scenarios by calculating the covariance matrix of galaxy ellipticities in four visual filters $(\mathrm{B}, \mathrm{V}, \mathrm{i}, \mathrm{z})$. We find that galaxy shapes are highly correlated, and estimate the gain in galaxy number density by combining their shapes.
\end{abstract}

Subject headings: cosmology:gravitational lensing

\section{Introduction}

Weak gravitational lensing surveys have become a powerful tool for testing cosmological theories. Until recently, the majority of weak lensing surveys have only used shape information from a single color. Some surveys such as the Sloan Digital Sky Survey (SDSS) 1 , the CFH Legacy survey $\sqrt[2]{2}$, COMBO-1 $\sqrt{3}$, and COSMOS4 have used multiple color information for estimating photometric redshifts (Sheldon et al. 2004; Semboloni et al. 2006; Kitching et al. 2007; Massey et al. 2007). Another use for multi-filter data has been as a cross check against systematic effects, since many systematics are expected to be color dependent, whereas the lensing signature is achromatic (Sheldon et al. 2004; Semboloni et al. 2006). However, almost no study to date has made systematic use of the shape information from images in the different colors with the goal of increasing the signal-to-noise of the shear information.

\footnotetext{
${ }^{1}$ http://www.sdss.org/

${ }^{2}$ http://www.cfht.hawaii.edu/Science/CFHLS/

${ }^{3}$ http://www.mpia.de/COMBO/combo_index.html

${ }^{4}$ http://cosmos.astro.caltech.edu/
} 
Future surveys, such as KIDS 5 , DES6 6 , PanStarrs 7 , SNAP 8 and LSST 9 will be obtaining better imaging data in multiple filters over much wider areas. Thus it behooves us to determine whether there is any significant advantage to using the shape information from the multiple filters in addition to the usual magnitude information.

There are two important regimes to consider with regards to this question. First, with bright objects that have measurable shapes in several colors, it might be possible to extract several independent shear estimators from the same physical galaxy. The plausibility of this idea comes from the fact that galaxies look different in different colors. For example, you can often see starbursting regions in bluer filters which are mostly unnoticeable in redder filters. Similarly, giant HII regions are only very bright in filters which includes rest frame $656 \mathrm{~nm}$. Thus a galaxy with such regions can look quite different in this filter than it does in other filters.

Case A: In the limiting case that galaxies look completely different in $N_{\mathrm{f}}$ different filters, we could obtain an independent estimate of the shear at the location of a galaxy from every filter with a measurable shape. In other words, we have an independent sampling of the shape noise in each filter. This leads to an effective increase of the number density of available galaxies by a factor of $N_{\mathrm{f}}$ : clearly this is a potentially large reduction in the statistical error in shear measurement.

Case B: The other limit is that the shapes are basically the same in every color. That is, the shape noise is sampled only once per galaxy, and we only get one shear estimator per galaxy. In this case we only obtain a reduction in measurement error, which is in any case smaller than the error due to the intrinsic ellipticities of galaxies. However if the galaxy shapes are the same in different filters, it is worth considering the set of galaxies for which a good measurement of its shape is possible using the data in multiple filters, where no such measurement is possible with the data in any one filter.

Since there are many more faint galaxies than bright galaxies, a large fraction of galaxies are only slightly too faint for a useful shape measurement. There are obviously more photons in multiple filters than in just one, so it is plausible that we could significantly increase the number of measured shapes by using the multi-filter data.

In $\oint 2$, we investigate which of these extremes is closer to the truth and what the implications of this would be. We investigate the possibile gain in effective number density of faint galaxies via multi-filter measurements in $\S 3$ and conclude in $\S 4$.

\footnotetext{
${ }^{5}$ http://www.astro-wise.org/projects/KIDS/

${ }^{6} \mathrm{http}: / / \mathrm{www}$. darkenergysurvey.org/

${ }^{7}$ http://pan-starrs.ifa.hawaii.edu/

${ }^{8}$ http://snal.lbl.gov/

${ }^{9}$ http://www.lsst.org/
} 


\section{Multi-color Shear Estimators from Bright Galaxies}

Here we consider Hubble Space Telescope data from the GOODS10 (Giavalisco et al. 2004) survey which makes comparably deep observations in four filters. We used the GOODS North data below, which cover 160 square arcminutes to depths of about 27th magnitude and had about 200,000 detected galaxies. The filters are B, V, i and $\mathrm{z}-\mathrm{a}$ fairly typical set of filters for an imaging survey. The limiting magnitudes (point source AB magnitude) in these filters are 27.8, 27.8, 27.1 and 26.6 respectively (Giavalisco et al. 2004).

For each galaxy, we assume that we can measure a shape in each of these filters, which we call $e_{B}, e_{V}, e_{i}$ and $e_{z}$, with measurement errors $\sigma_{B}^{2}, \sigma_{V}^{2}, \sigma_{i}^{2}$ and $\sigma_{z}^{2}$ respectively.

Throughout this paper, we assume that the shape measurements have been converted into an unbiased shear estimator. That is, they have been corrected for the shear polarizability or responsivity. That is, the expectation value of $e$ is $\gamma=\gamma_{+}+i \gamma_{\times}$. In this study, we used a fairly simple PSF correction, which should be more than sufficient for these purposes, but real applications would want to use a sophisticated PSF removal technique (e.g. Hoekstra et al. 1998; Bernstein \& Jarvis 2002; Refregier \& Bacon 2003).

If the intrinsic shapes were completely independent in different filters, these four shape measurements could give us four independent estimates of the gravitational shear field at the location of the galaxy. On the other hand, if the intrinsic shapes were identical in each filter, then these four measurements would only give us one shear estimator with a smaller effective measurement error. We use the GOODS data to see which scenario is closer to what we can expect for a real survey.

For a single-filter shear estimator, $\hat{\gamma}=e$, the estimate of the noise on this quantity is

$$
\operatorname{Var}(\hat{\gamma})=\left\langle|e|^{2}\right\rangle+\sigma^{2}
$$

The generalization of this equation to the multi-filter case is:

$$
\begin{aligned}
\hat{\gamma} & =\left(\begin{array}{c}
e_{B} \\
e_{V} \\
e_{i} \\
e_{z}
\end{array}\right) \\
\operatorname{Cov}(\hat{\gamma})= & C+\Sigma \\
= & \left(\begin{array}{cccc}
\left\langle e_{B} e_{B}^{*}\right\rangle & \left\langle e_{B} e_{V}^{*}\right\rangle & \left\langle e_{B} e_{i}^{*}\right\rangle & \left\langle e_{B} e_{z}^{*}\right\rangle \\
\left\langle e_{V} e_{B}^{*}\right\rangle & \left\langle e_{V} e_{V}^{*}\right\rangle & \left\langle e_{V} e_{i}^{*}\right\rangle & \left\langle e_{V} e_{z}^{*}\right\rangle \\
\left\langle e_{i} e_{B}^{*}\right\rangle & \left\langle e_{i} e_{V}^{*}\right\rangle & \left\langle e_{i} e_{i}^{*}\right\rangle & \left\langle e_{i} e_{z}^{*}\right\rangle \\
\left\langle e_{z} e_{B}^{*}\right\rangle & \left\langle e_{z} e_{V}^{*}\right\rangle & \left\langle e_{z} e_{i}^{*}\right\rangle & \left\langle e_{z} e_{z}^{*}\right\rangle
\end{array}\right)+\left(\begin{array}{cccc}
\sigma_{B}^{2} & 0 & 0 & 0 \\
0 & \sigma_{V}^{2} & 0 & 0 \\
0 & 0 & \sigma_{i}^{2} & 0 \\
0 & 0 & 0 & \sigma_{z}^{2}
\end{array}\right)
\end{aligned}
$$

If the matrix $C$ is close to diagonal, then we are in the first case described above which gives us four

\footnotetext{
${ }^{10}$ http://www.stsci.edu/science/goods/
} 
independent shear estimates. However, if the 16 values in $C$ are all of similar magnitude, then we are in the second case, which implies that each galaxy only gives us a single effective shear estimate.

The GOODS data include galaxies to a limiting i magnitude of about 27, with a median i magnitude of 25.75. Requiring galaxies to be observed in all four filters lowers the median i magnitude to 25.4, not too much shallower than the original data set. We measured the shape in each filter and the corresponding uncertainties using a simplified version of the techniques described in Bernstein \& Jarvis (2002).

Using this data, we calculate the matrix $C$ to be

$$
\begin{aligned}
C & =\left\langle\hat{\gamma} \hat{\gamma}^{\dagger}-\Sigma\right\rangle \\
& =\left(\begin{array}{llll}
0.25 & 0.21 & 0.20 & 0.18 \\
0.21 & 0.22 & 0.20 & 0.19 \\
0.20 & 0.20 & 0.22 & 0.18 \\
0.18 & 0.19 & 0.18 & 0.22
\end{array}\right)
\end{aligned}
$$

Note that, while the shapes are intrinsically complex numbers, and therefore $C$ is as well, the imaginary components of this matrix turn out to be insignificant (i.e. consistent with 0 ). In fact, this is necessarily the case in an isotropic universe. Therefore we treat the matrix as effectively real to avoid the clutter of the imaginary components.

This matrix is evidently closer to our second case, where all 16 elements in the matrix have a similar value. However, we can quantify this statement by diagonalizing the matrix:

$$
\begin{aligned}
\boldsymbol{\Lambda} & =V^{T} C V \\
& =\left(\begin{array}{cccc}
0.81 & 0 & 0 & 0 \\
0 & 0.05 & 0 & 0 \\
0 & 0 & 0.03 & 0 \\
0 & 0 & 0 & 0.02
\end{array}\right)
\end{aligned}
$$

This gives the noise matrix in a new basis where the measurements are uncorrelated. The net signal-to-noise for these four uncorrelated estimators is given by

$$
\gamma_{\operatorname{cosm}}^{T} V \Lambda^{-1 / 2}=\left(\begin{array}{llll}
2.2 & 0.28 & 0.07 & 0.06
\end{array}\right)
$$

where $\gamma_{\operatorname{cosm}}^{T}=\left(\begin{array}{llll}1 & 1 & 1 & 1\end{array}\right)$ is the cosmological lensing signal in the original basis.

When combining all of the shear data, these signal-to-noise values add in quadrature, leading to a net increase of less than $1 \%$ over using only the first component. Therefore, we conclude that there is effectively no extra shear information available per galaxy from using the shapes measured in multiple filters.

In general the shapes of spiral and irregular galaxies vary more with color than ellipticals, so perhaps this technique might be worthwhile for this subset of the galaxies. To test this, we divided the galaxy sample in half according to V-I color. The blue half, which should have more early type galaxies, did show more 
variation than the red half, but the net signal-to-noise increase was still less than $2 \%$ relative to using only the first component. So the potential improvement for the blue galaxies is still insignificant. A similar test based on a concetration parameter was even less effective.

Note that the measurement error (in contrast to the shape error we have been discussing above) in the shear estimate of each galaxy can be reduced by using the dominant eigenvalue. The net measurement error

can be reduced by at most $\sqrt{N_{\mathrm{f}}}$, where $N_{\mathrm{f}}$ is the number of filters, 4 in this case. However, in most cases, the measurement errors will be different in each of the four filters, so the improvement over the best-measured shape will be somewhat less than this.

Furthermore, one typically cannot obtain a usable shape measurement with measurement noise $\sigma>$ 0.1 . As this is already well below the value of the shape noise, even reducing the measurement noise by a full factor of two will not greatly improve the uncertainties in the final shear statistics.

\section{Multi-color Shear Estimators from Faint Galaxies}

The other case where data in multiple colors could be helpful for weak lensing shear estimates is when the data in any one filter is too noisy to obtain a useful shape measurement, but where the total signal-tonoise from all the filters would allow a useful measurement to be obtained.

The goal here is to increase the total number density of galaxies in the survey, which directly increases the signal-to-noise of the final weak lensing data product. We want to know by how much we can increase the effective number density.

We will use the rule of thumb that one needs a measurement error $\sigma<0.1$ to obtain a useful shear estimate. Galaxies with a smaller signal-to-noise, which would have a measurement error larger than 0.1 generally do not converge, or have some other problem which makes the estimate unreliable. The exact value of 0.1 is unimportant for our purpose. Indeed, one can also increase the number density by finding a way to increase this number while still obtaining reliable unbiased shear estimates. Regardless of what number we choose, the relative gain of using multi-filter data versus just the best filter for each galaxy should be roughly similar.

In each filter, $k$, the galaxy has the following measured values: the flux, $F_{k}$, measured in photons, the sky noise $n_{k}$, measuring the variance per square arcsec, the radius, $r_{k}$, of the best fit Gaussian, and the PSF radius, $r_{\mathrm{PSF}, k}$. In terms of these values, the measurement uncertainty in the shear estimator in a single filter is:

$$
\sigma_{k}=\frac{\sqrt{n_{k} \pi r_{k}^{2}+F_{k}}}{F_{k}} \frac{r_{k}^{2}}{r_{k}^{2}-r_{\mathrm{PSF}, k}^{2}} .
$$

The first factor here is simply the Poisson photon noise in the image divided by the flux. For ground-based images, the noise is dominated by the sky noise, so the second term in the numerator is negligible. The second factor accounts for the dilution effect (Bernstein \& Jarvis 2002), also known as smear polarizability in the KSB method (Kaiser. Squires, \& Broadhurst 1995). Basically, the PSF rounds the image of a galaxy, 
so the intrinsic ellipticity estimate is always larger than the measured observed ellipticity. The measurement uncertainty, $\sigma$, is also larger by the same factor.

The measurement error on a combined multi-filter shear estimate is:

$$
\sigma_{\text {multi }}=\left(\sum_{k}^{N_{\mathrm{f}}} \frac{1}{\sigma_{k}^{2}}\right)^{-1 / 2} .
$$

In practice, we do not simply average the individual measurements from the $N_{\mathrm{f}}$ filters, as this equation might imply. Instead, we perform a global maximum likelihood fit to the intrinsic shape of the galaxy. The observations each have a different PSF size and shape, so the calculations must take into account how the different PSF's have affected the instrinsic shape. A more thorough description of this process can be found in Nakajima \& Bernstein (2007). However, for the purposes of this paper, Equation 11] suffices for our estimates of $\sigma_{\text {multi }}$.

To obtain a simplified estimate of the gain possible with $N_{\mathrm{f}}$ filters, consider a galaxy population described by the number magnitude relation at the flux limit

$$
N(F) \propto F^{-2.5 s}
$$

where $F$ is the flux in a given filter (we have dropped the subscript $k$ here). Note that $s$ is more commonly known as the slope of the (log of) the number counts versus limiting magnitude. For visible filters and galaxies at $z \gtrsim 1$ current data show that $s \lesssim 0.4$, giving $N \propto F^{-1}$ or shallower. If we assume that $\sigma_{k} \equiv \sigma$ is the same in all filters, we get $\sigma_{\text {multi }}^{2}=\sigma^{2} / N_{\mathrm{f}} \sim 1 /\left(F^{2} N_{\mathrm{f}}\right)$ (approximating from equation 10). Thus using $N_{\mathrm{f}}$ filters lowers the effective flux limit to $F / \sqrt{N_{\mathrm{f}}}$, which corresponds to an increase in number density of $n \rightarrow n N_{\mathrm{f}}^{2.5 s / 2}$. For $s \simeq 0.4$, the increase in $n$ is a factor of $\sqrt{N_{\mathrm{f}}}$; it is lower for smaller $s$. Thus a rough guide to the maximum gain possible from multiple filters is that by using four filters with comparable signal-to-noise shape measurements, the effective number density of galaxies doubles.

For the GOODS data we estimate how much the number density can be increased by using the galaxies which have $\sigma_{\text {multi }}<0.1$, but for which all $\sigma_{k}>0.1$. We also impose an additional restriction, which we have not discussed in this paper, but which experience has shown is required for unbiased shear estimates. Namely, we only use galaxies which have $r>1.2 r_{\mathrm{PSF}}$. When this criterion gives different answers in different filters, we do consider the data from those filters which pass the test (i.e., we do not reject a galaxy entirely just because the PSF is too large in one of the filters.)

Using the GOODS data, we find that the number density increases by $30 \%$ if one uses the multi-filter shear estimates rather than only those with a measurable shear in at least one filter. For this data set, most of the galaxies with measurable shapes in any filter were measureable in the z filter. In fact if the multi-filter shapes are compared to the shape measured just in the $\mathrm{z}$ filter the net increase in number density is $34 \%$, only slightly higher (it would of course be significantly higher if the comparison was made with any of the other filters). This increase however is signficantly smaller than the idealized estimate of a factor of two above, because the measurement error is far from constant in the different filters. This is mostly due to the galaxy spectrum being far from flat; the $\mathrm{z}$ filter was the shallowest of the four in terms of AB magnitude, but 
it still typically had the highest signal to noise. For shallower surveys, such as planned near-term surveys from the ground, the median redshifts will be lower. Hence one would expect that the signal to noise will peak at lower wavelengths, but the effective number of filters will probably still be at most 2 .

The improvement is likely to be greater for surveys with more than four, presumably narrower, filters and for specific galaxy populations. It does not appear to be much larger based on tests with simulated images kindly provided by D. Johnston which used realistic shapelet models for the galaxy shapes based on UDF images. For these data, we found that the gain in galaxy number density with six narrow filters was still about $30 \%$.

A second factor that can provide benefits is that the fainter galaxies are at higher redshift than the median for the whole sample. Hence they carry greater lensing signal and offer a wider redshift range for tomography. Therefore the increase in number density may underestimate the resulting increase in signalto-noise for cosmological parameter estimation.

We also note that we assumed that the lower limit in $\sigma$ from the multi-filter data is the same as for single filter data. This sounds like an obvious assumption, but in fact it is not yet true. Current algorithms for measuring a shape from multiple images (whether the same or different filters) have more trouble converging than algorithms which only use data from a single image (keeping total signal-to-noise constant). We expect that it should be possible to improve these algorithms to where the multi-image algorithm is as effective as the single-image algorithm, but it does require further work.

\section{Discussion}

We found based on the sample of galaxies from GOODS that galaxy shapes are highly correlated between visual filters. Hence there is no significant advantage to having multi-filter shape information for bright galaxies in a weak lensing survey. However, for the fainter galaxies that are close to the signal-tonoise threshold, we find that there is a significant gain in using multiple filters because one can measure shapes for galaxies below the threshold in any one filter. There are 34\% more galaxies with usable shear estimates in the GOODS B,V,i,z data by combining shape information from all four filters than by simply using shapes measured in $\mathrm{z}$, the single best filter for all galaxies.

The specific results are somewhat tied to the properties of the GOODS data set. For surveys which have more filters or which have a different magnitude limit or noise properties, the exact benefit may vary. For ground based surveys the sky noise and seeing will play a role as well. However, based in part on tests with simulated images, we believe that the basic result will hold - there are improvements to be had due to an increase in galaxy number density at the faint end, but they are below the factor of two (or more) one might have expected in an idealized case. The galaxy properties underlying this conclusion are: (i) galaxy shapes are highly correlated across the visual filters, but, (ii) the signal-to-noise for shape measurements varies so that only a few filters contribute significantly for a given galaxy.

There is an additional gain in lensing measurements because the faint galaxies recovered using multi- 
filter shapes are at higher redshifts than the median redshift of the whole sample. These galaxies carry greater lensing signal, and more usefully, enable more tomographic bins to be used for cosmological analysis. Surveys of modest depth, for which these faint galaxies lie at redshifts $\gtrsim 1$, will have the greatest improvment in accuracy of parameters such as the dark energy equation of state. The key factors that impact the gain for a particular survey are: the redshift distribution of the galaxy sample, the typical galaxy sizes relative to the seeing at the faint end, and the signal-to-noise per filter needed for the desired accuracy in photo-z's (the photo-z measurement is not considered in this study).

Finally, we emphasize that systematic errors can be checked and partially eliminated with multi-filter data. The SDSS analyses of galaxy-galaxy lensing (Fisher et al. 2000) used the consistency of the tangential shear profile in the SDSS g', r' and i' filters as one of the main checks of systematics. In future surveys that aim to measure shears with percent level accuracy, comparison of shape measurements will be valuable in checking for the effect of color dependent PSFs - these affect stars and galaxies differently and are not well quantified at the present time.

We thank Gary Bernstein, Sarah Bridle, Dave Johnston, Jason Rhodes, Fritz Stabenau, Masahiro Takada, Ludo van Waerbeke and David Wittman for helpful discussions. We also thank the anonymous referee for very useful feedback. This work is supported in part by NSF grant AST-0607667, the Department of Energy and the Research Corporation. 


\section{REFERENCES}

Bernstein, G. M., \& Jarvis, M. 2002, AJ, 123, 583

Fischer, P. et al. 2000, AJ, 120, 1198

Giavalisco, M., et al. 2004, ApJ, 600, L93

Hoekstra, H., Franx, M., Kuijken, K., \& Squires, G. 1998, ApJ, 504, 636

Kaiser, N., Squires, G., \& Broadhurst, T. 1995, ApJ, 449, 460

Kitching, T. D., Heavens, A. F., Taylor, A. N., Brown, M. L., Meisenheimer, K., Wolf, C., Gray, M. E., \& Bacon, D. J. 2007, MNRAS, 376, 771

Massey, R., et al. 2007, ApJS, 172, 239

Nakajima, R., \& Bernstein, G. 2007, AJ, 133, 1763

Refregier, A. \& Bacon, D. 2003, MNRAS, 338, 48

Semboloni, E., et al. 2006, A\&A, 452, 51

Sheldon, E. S., et al. 2004, AJ, 127, 2544 